\title{
Medical resource utilization for administration of trastuzumab in a New Zealand oncology outpatient setting: a time and motion study
}

This article was published in the following Dove Press journal:

ClinicoEconomics and Outcomes Research

29 July 2015

Number of times this article has been viewed

\author{
Richard T North' \\ Vernon J Harvey ${ }^{2}$ \\ Levonne C Cox ${ }^{2}$ \\ Stuart N Ryan ${ }^{3}$ \\ 'Cancer and Haematology Service, \\ Tauranga Hospital, Tauranga, ${ }^{2}$ Regional \\ Cancer and Blood Centre, Auckland \\ City Hospital, Auckland, ${ }^{3}$ Medical \\ Affairs, Roche Products (New \\ Zealand) Ltd, Auckland, New Zealand
}

Background: In New Zealand, trastuzumab is standard therapy for human epidermal growth factor receptor-2 (HER2)-positive early and metastatic breast cancer. Given the requirement for ongoing adjuvant or maintenance treatment and intravenous (IV) delivery, such a regimen consumes considerable health care resources. The development of a subcutaneous (SC) trastuzumab formulation with a short administration time offers the potential to reduce hospital expenditure. The aim of this study was to determine medical resource utilization associated with administration of trastuzumab SC injection via handheld syringe vs trastuzumab IV infusion in patients with HER2-positive breast cancer in New Zealand.

Methods: This noninterventional, descriptive study was conducted at the outpatient oncology centers at Auckland City and Tauranga Hospitals. Trained observers recorded times associated with health care professional (HCP) tasks and consumables use associated with preparation and administration of trastuzumab IV or SC in women with early or metastatic breast cancer. The cost for each formulation was calculated as the mean cost of HCP time (based on Pharmaceutical Management Agency hourly rates) plus the mean cost of consumables used.

Results: Use of trastuzumab SC vs IV reduced mean chair time by 36.95 minutes and total nurse time by 6.12 minutes; there was a 20.45 -minute reduction in pharmacist time when the SC formulation was used. After adding consumable costs, the overall estimated saving with trastuzumab SC vs IV was $\$ 76.94$ (New Zealand dollars) per patient per cycle.

Conclusions: Compared with trastuzumab IV infusion, administration of trastuzumab via SC injection reduced time spent in the clinic and decreased HCP resources and consumables needed to administer treatment. These reductions could contribute to a decrease in health care costs and an improvement in the efficiency of HER2-positive breast cancer treatment delivery.

Keywords: breast cancer, trastuzumab, subcutaneous, resource use, cost analysis

\section{Background}

Breast cancer is the second most common cancer in New Zealand and the third leading cause of cancer death. ${ }^{1}$ In 2011 , the most-recent full year for which statistics are available, 2,867 new cases of breast cancer and 636 deaths were reported. ${ }^{2}$ Approximately $18 \%$ of breast cancers in New Zealand are human epidermal growth factor receptor-2 (HER2)-positive. ${ }^{3}$

Trastuzumab is standard treatment for HER2-positive early breast cancer (eBC) and metastatic breast cancer $(\mathrm{mBC})$, being administered as an intravenous (IV) infusion, in part with chemotherapy, as an every-3-weeks regimen for 12 months in eBC (17 cycles), or until disease progression in $\mathrm{mBC}$. Trastuzumab IV needs to be reconstituted into solution for infusion, with the dose calculated on the basis of patient bodyweight. ${ }^{4}$ IV trastuzumab treatment starts with a loading dose infused
Correspondence: Stuart N Ryan Country Medical Manager, Medical Affairs, Roche Products (New Zealand) Ltd, 98 Carlton Gore Road, Newmarket 0123, Auckland, New Zealand

Tel +64095239403

Fax +64095239483

Email stuart.ryan@roche.com 
over 90 minutes, followed by subsequent maintenance doses infused over 30 minutes. $^{4}$

Given the requirement for up to 12 months of adjuvant or maintenance treatment, such a regimen consumes considerable health care resources, including drug preparation and administration, clinic and chair time, and physician time dedicated to patient interaction. ${ }^{5}$ Furthermore, insertion of an indwelling venous catheter is common in patients requiring prolonged IV infusion therapy, adding the costs of theatre and anesthetist time; complications such as infection or thrombosis ${ }^{6,7}$ may also be associated with additional costs in some cases.

A subcutaneous (SC) formulation of trastuzumab was developed to allow drug administration over a shorter time period, with the goal of improving convenience and compliance, particularly during long-term therapy. The ability to do this was facilitated by the fact that trastuzumab has a wide therapeutic window ${ }^{8}$ with no known dose-limiting toxicities. ${ }^{9}$

SC administration of volumes $>1 \mathrm{~mL}$ has been limited by the structure and physiology of the SC layer, which contains a matrix of hyaluronan and collagen fibers. ${ }^{8}$ To overcome this issue, the trastuzumab SC formulation includes recombinant human hyaluronidase as a novel excipient. ${ }^{10}$ Hyaluronidase hydrolyses hyaluronan, decreases resistance of the interstitial matrix ${ }^{11,12}$ and degrades the extracellular matrix. This allows monoclonal antibodies to diffuse through the channels, ${ }^{12}$ thereby facilitating SC administration of larger volumes of fluid and improving delivery of SC drugs to the systemic circulation. The effects of recombinant human hyaluronidase are temporary and reversible within 24 hours. ${ }^{12}$

Trastuzumab SC can be administered over approximately 5 minutes, a much shorter period of time than that required for the trastuzumab IV loading and maintenance doses. ${ }^{4,10}$ In addition, trastuzumab SC is given as a fixed dose that does not require adjustment based on bodyweight. ${ }^{10}$

Pharmacokinetic modeling, based on data from an openlabel Phase I/IB study, ${ }^{13}$ determined that a $600 \mathrm{mg}$ fixed dose of trastuzumab SC provided drug exposure that was comparable to the IV trastuzumab regimen, without the need for a loading dose. This dose of trastuzumab SC was subsequently tested in the Phase III HannaH study, which showed that trastuzumab SC was noninferior to trastuzumab IV with respect to efficacy (pathological complete response rate) and had an equivalent tolerability profile. ${ }^{14}$

One of the key principles guiding the New Zealand Ministry of Health Medical Oncology National Implementation Plan ${ }^{15}$ is to "effectively, equitably, and sustainably meet the future demand for medical oncology services, given the significant workforce and resource constraints that exist". A second aim is to "maintain high quality of care and improve the quality of life for people with cancer". The possibility of delivering trastuzumab over a shorter duration closely aligns with these principles. It also offers the potential to move treatment delivery to the outpatient setting and away from infusion services that are facing challenges due to increased demand for complex treatments. ${ }^{15}$

The aim of this study was to determine medical resource utilization associated with administration of trastuzumab SC injection compared with trastuzumab IV infusion in patients with HER2-positive breast cancer in New Zealand.

\section{Methods \\ Study design}

This noninterventional, descriptive study was conducted alongside the ongoing SafeHer clinical trial (NCT01566721), a study investigating the safety and tolerability of trastuzumab SC via handheld syringe or single-injection device. The time and motion study was conducted at the outpatient oncology centers at Auckland City Hospital (trastuzumab IV) and Tauranga Hospital (trastuzumab SC and IV).

\section{Subjects}

Patients receiving trastuzumab SC via handheld syringe were recruited by the health care professionals (HCPs) who were administering trastuzumab to participants in the SafeHer clinical trial at Tauranga Hospital. Patients with eBC were approached during a routine visit from March 2013 to May 2013 as part of their enrolment in SafeHer and given information about the time and motion study by a member of the clinical team. Prior to trastuzumab administration, patient consent was sought for an observer to be present during that visit. Patients receiving trastuzumab IV were being treated in the metastatic and adjuvant settings and were undergoing routine clinical care in the outpatient clinic of either Tauranga or Auckland Hospital in April 2013 and May 2013. At the start of the clinic visit they were given information about the time and motion study by the HCP who would be administering trastuzumab. Under the same protocol as the SC group, patient consent to allow an observer to be present during their clinic visit was sought prior to trastuzumab administration.

This study was an audit that does not involve the use, collection, or storage of human tissue, and therefore the Health and Disability Ethics Committees advised that the study did not require their review. 


\section{Data collection}

Active HCP time captured during the time and motion study related to tasks associated with administration of trastuzumab SC or IV, as detailed below.

New Zealand-specific standardized Treatment Observation Forms were created, based on those used in a time and motion study conducted in the United Kingdom. ${ }^{16}$ Times for entering the bed/chair, starting trastuzumab treatment, stopping trastuzumab treatment, and exiting the bed/chair were recorded in the Treatment Observation Form in hours and minutes (hh:mm) by a single trained observer. A stopwatch was used by the same observer to record times for active involvement in specific trastuzumab treatment-related tasks as follows: install venous catheter/line flushing; prepare and administer premedication (if required); prepare for trastuzumab administration in the care unit; initiate trastuzumab therapy; monitor patient during treatment; disconnect infusion/flush infusion line/dispose of materials (IV only); monitor patient after treatment.

Other variables recorded on the same Treatment Observation Form were type of IV access (IV only), the occurrence of any unexpected events that disrupted typical patient flow, use and type of premedication, and consumables used for each task (including the exact product and the number of units used).

Drug preparation time was also documented by an observer using a stopwatch. Trastuzumab for SC injection via handheld syringe was prepared by nurses in the clinic. Trastuzumab solution for IV infusion was prepared in the hospital pharmacy, where the total time was calculated as the time from taking the prescription to leaving the prepared solution for collection. Specific timed tasks for trastuzumab IV preparation were: prepare and label; add trastuzumab and mix with diluent; stand vial and check for impurities; calculate and check dose; add to infusion and mix; finalize for release. Consumables used during the preparation and delivery of both formulations were noted on the Treatment Observation Form.

Costs for indwelling venous lines (eg, Portacath, Hickman, and Groshong) were not included in cost calculations because patients who required or had these access devices in place had had them inserted for previous chemotherapy.

Unit costs for HCP and personnel time were derived from Pharmaceutical Management Agency (PHARMAC) published costs, and were \$45 NZD (New Zealand dollars) per hour for a registered nurse, $\$ 50$ NZD per hour for a hospital pharmacist, and \$65 NZD per hour outpatient bed per chair cost. ${ }^{17}$

\section{Cost model}

A simple cost-minimization model was developed to estimate cost savings associated with delivery of trastuzumab $\mathrm{SC}$ compared with trastuzumab IV in the outpatient setting. All outpatient consumables and times directly related to both routes of administration were quantified from the standardized Treatment Observation Forms. Average estimates for delivery of trastuzumab IV and trastuzumab SC were calculated for all hospital and pharmacy data. These data were used to estimate resource inputs, which were then applied to the PHARMAC unit cost data for hourly rates and to consumable costs to allow determination of the estimated difference in costs between the two treatment groups. Only one trastuzumab IV formulation is available in New Zealand (Herceptin ${ }^{\circledR}$, Roche Products Ltd, Auckland, New Zealand). It was assumed that drug acquisition costs for trastuzumab IV and trastuzumab SC would generally be equivalent, and therefore these were not included in the analysis.

\section{Results}

A total of six patients receiving trastuzumab SC as part of the ongoing SafeHer clinical trial, and 12 patients receiving routine clinical treatment with trastuzumab IV (six each in Tauranga and Auckland) consented to allow an observer to record data for the time and motion study during their treatment visit and were included in the final analysis.

Administration of trastuzumab SC reduced nursing time by an average of $43 \%$ and chair time by an average of $75 \%$ compared with trastuzumab IV (Table 1). Although the two trastuzumab formulations were prepared in different settings (IV by the pharmacy and SC by the clinic nurse), the HCP time dedicated to drug preparation was also substantially lower for trastuzumab SC (Table 1).

Usage of consumables was low in both the trastuzumab $\mathrm{SC}$ and IV groups, but some savings were associated with the use of trastuzumab SC (Table 2).

Overall time to prepare and deliver trastuzumab therapy was markedly lower for the SC vs IV formulation (Table 1). Average HCP nurse time, chair cost, and pharmacist time savings associated with the use of trastuzumab SC injection compared with trastuzumab IV infusion were estimated to be $\$ 61.67$ NZD per administration (Table 1), and consumables cost savings were estimated as \$15.27 NZD per administration, with the largest single item being the cost of the delivery line (note that costs for indwelling venous lines were not included) (Table 2). Thus, estimated cost saving achieved by switching to the SC formulation for delivery of trastuzumab in the New Zealand District Health Board 
Table I Times and costs associated with a single administration of trastuzumab SC vs IV

\begin{tabular}{|c|c|c|c|c|c|}
\hline & \multicolumn{2}{|l|}{ Trastuzumab } & \multirow{2}{*}{$\begin{array}{l}\text { Difference } \\
\text { between means } \\
\text { (SC - IV) }\end{array}$} & \multirow{2}{*}{$\begin{array}{l}\text { Cost } \\
\text { per hour } \\
\text { (NZD) }\end{array}$} & \multirow{2}{*}{$\begin{array}{l}\text { Cost savings, } \\
\text { SC vs IV } \\
\text { (NZD) }\end{array}$} \\
\hline & IV & SC & & & \\
\hline \multicolumn{6}{|l|}{ Treatment (minutes) } \\
\hline Time spent in chair & $47.44(42.78 ; 41.12-73.15)$ & $10.49(10.05 ; 8.22-13.28)$ & -36.95 & 65.00 & 40.03 \\
\hline Duration of trastuzumab delivery & $37.60(32.55 ; 31.90-62.45)$ & $5.67(5.60 ; 5.42-6.02)$ & -31.93 & & \\
\hline Total HCP nurse time & $13.02(12.97 ; 10.18-16.12)$ & $6.90(6.90 ; 6.65-7.21)$ & -6.12 & 45.00 & 4.59 \\
\hline \multicolumn{6}{|l|}{ Drug preparation (minutes) } \\
\hline Prepare and label & 5.50 & 0 & -5.50 & & 4.58 \\
\hline Add and mix with diluent & 1.63 & 0 & -1.63 & & 1.36 \\
\hline Stand vial and check for impurities & 5.62 & 0 & -5.62 & & 4.68 \\
\hline Calculate and check dose & 2.22 & 0 & -2.22 & & 1.85 \\
\hline Add to infusion and mix & 4.47 & 0 & -4.47 & & 3.73 \\
\hline Finalize for release & 1.02 & 0 & -1.02 & & 0.85 \\
\hline Total pharmacist time & $20.45(20.45 ; 20.28-20.60)$ & 0 & -20.45 & 50.00 & 17.05 \\
\hline Overall total & & & & & 61.67 \\
\hline
\end{tabular}

Note: Mean values are reported, with median and range in parentheses where available.

Abbreviations: IV, intravenous; SC, subcutaneous; NZD, New Zealand dollars; HCP, health care professional.

outpatient oncology clinic setting was \$76.94 NZD per patient per cycle.

\section{Discussion}

The results of this descriptive time and motion study suggest that switching to trastuzumab SC injection from trastuzumab IV infusion in a New Zealand oncology outpatient treatment setting decreases trastuzumab treatmentrelated tasks in the care unit, drug preparation time, chair time, and the volume and cost of consumables.

Although differences between health care systems limit the generalizability of results, these New Zealand data are similar to those obtained in Europe ${ }^{18}$ and the United Kingdom ${ }^{16}$ showing reduced chair time and HCP resource

Table 2 Mean consumables use and costs associated with a single administration of trastuzumab SC vs IV

\begin{tabular}{|c|c|c|c|c|c|}
\hline & \multicolumn{2}{|c|}{ Trastuzumab } & \multirow{2}{*}{$\begin{array}{l}\text { Difference between } \\
\text { means, SC - IV }\end{array}$} & \multirow{2}{*}{$\begin{array}{l}\text { Cost per } \\
\text { item (NZD) }\end{array}$} & \multirow{2}{*}{$\begin{array}{l}\text { Estimated cost savings, } \\
\text { SC vs IV (NZD) }\end{array}$} \\
\hline & IV & SC & & & \\
\hline \multicolumn{6}{|l|}{ Consumables (units) } \\
\hline $5 \mathrm{~mL}$ or $10 \mathrm{~mL}$ BD syringe & 2 & I & -1 & 0.25 & 0.25 \\
\hline $20 \mathrm{~mL}$ or $26 \mathrm{~mL}$ precision glide needle & 3 & 2 & -1 & 0.25 & 0.25 \\
\hline First-aid plastic strip & I & 1 & 0 & 0.10 & 0 \\
\hline Multisorb squab & 2 & I & -1 & 0.10 & 0.10 \\
\hline Gown & I & 1 & 0 & 0.50 & 0 \\
\hline Alcohol wipe & 4 & 1 & -3 & 0.01 & 0.03 \\
\hline Gloves & 2 & 1 & -1 & 0.20 & 0.20 \\
\hline Mask & 0 & 1 & +1 & 0.15 & -0.15 \\
\hline Tray & 0 & I & +1 & 0.15 & -0.15 \\
\hline IV line & I & 0 & -1 & 9.90 & 9.90 \\
\hline Total consumables & & & & & 10.43 \\
\hline \multicolumn{6}{|l|}{ Pharmacy consumables (units) } \\
\hline $\mathrm{BD}$ syringe & 2 & 0 & -2 & 0.25 & 0.50 \\
\hline Needle & 2 & 0 & -1 & 0.25 & 0.50 \\
\hline Mask & I & 0 & -1 & 0.15 & 0.15 \\
\hline Gown & I & 0 & -1 & 0.50 & 0.50 \\
\hline Alcohol wipe & 4 & 0 & -4 & 0.01 & 0.04 \\
\hline Gloves & 2 & 0 & -2 & 0.20 & 0.40 \\
\hline Infusion bag ( $250 \mathrm{~mL}$ sodium chloride) & I & 0 & -1 & 2.75 & 2.75 \\
\hline Total pharmacy & & & & & 4.84 \\
\hline Overall total & & & & & 15.27 \\
\hline
\end{tabular}

Abbreviations: BD, Becton, Dickinson and Company; IV, intravenous; SC, subcutaneous; NZD, New Zealand dollars. 
requirement in patients treated with trastuzumab SC compared with trastuzumab IV.

The European and UK data were obtained in patients being treated with the two different trastuzumab formulations as part of the PrefHer trial, ${ }^{19}$ a global, randomized, crossover preference study conducted in patients with eBC. In Europe, using estimates of the typical number of eBC patients being treated in the participating centers, total estimated savings in chair time associated with switching to trastuzumab SC ranged from 44-90 8-hour days annually. In addition, there was an associated $21 \%-52 \%$ reduction in the time HCPs spent actively involved in administering treatment. $^{18}$

Patients treated with trastuzumab SC in the United Kingdom also spent considerably less time in the care unit (30.3 minutes vs 94.5 minutes) and infusion chair (19.8 minutes vs 75.0 minutes) than did those receiving trastuzumab IV. ${ }^{16}$ In addition, mean HCP time was markedly lower when the SC formulation was used (24.6 minutes vs 92.6 minutes for IV administration), and consumable costs were lower (£2.94 vs $£ 8.81$, respectively). ${ }^{16}$ It should be noted that the latter study included total infusion duration in HCP time, as opposed to the European study, which considered only active patient monitoring during infusion. On the basis of the results of the UK study, it was concluded that switching from trastuzumab IV to the SC formulation could substantially reduce overall treatment costs. ${ }^{16}$ These findings are similar to those documented after a switch from IV to SC rituximab in non-Hodgkin lymphoma patients in the United Kingdom, which was also associated with reduced active $\mathrm{HCP}$ and chair time and lower costs. ${ }^{20}$

In a separate trastuzumab analysis, HCPs from a subset of PrefHer centers were asked to rate a variety of factors on their ability to impact cancer center efficiency and time required for trastuzumab administration. ${ }^{21}$ The biggest perceived impact of switching to trastuzumab SC, according to care unit respondents, was a reduced waiting list for any IV treatment at the infusion unit, followed by the potential for a more-flexible treatment schedule and the ability to treat more patients in the infusion unit. ${ }^{19}$ Respondents perceived that a reduction in dosing errors, reduced drug wastage, and increased staff availability for other tasks would be the main impacts of switching from trastuzumab IV to SC. ${ }^{15}$

Factors such as reduced waiting times and the flexibility to deliver treatment outside the infusion clinic offer the possibility of developing new patient care pathways defining when, where, how, and by whom trastuzumab therapy is administered. A switch to trastuzumab SC fits well with the new medical oncology model of care described by the New Zealand Ministry of Health, which includes identification of patient care pathway tasks and functions that can be performed by staff other than senior medical officers. ${ }^{15}$

In this study, trastuzumab SC was prepared by a registered nurse prior to administration to the patient. This was considered to be the most likely way that trastuzumab SC would be prepared outside of a clinical trial situation or on a ward. Should trastuzumab SC become available for general use within an oncology outpatient setting, preparation of the treatment dose may take place in the pharmacy or in the outpatient clinic depending on the preference or protocol of the individual hospitals. However, regardless of which HCP prepares trastuzumab SC prior to administration, it is reasonable to assume that the time savings achieved would be similar to those observed during this study.

Although New Zealand time and motion data from the current study were consistent with those obtained in other trials, it should be noted that these preliminary assessments of the impact of switching from trastuzumab IV to the SC formulation are likely to underestimate the potential time and cost savings. There are a number of reasons for this.

Firstly, the costs of indwelling IV catheter insertion and maintenance, and of managing complications, were not included in the analysis. Vascular access devices are one of the most common causes of health care-associated infections, including sepsis. ${ }^{22}$ The rate of sepsis after implantation of a venous access system for chemotherapy in a New Zealand population was $11 \% .^{7}$ Catheter-related sepsis indirectly affects outcomes of chemotherapy by compromising dose intensity, ${ }^{23}$ is associated with significant morbidity and increased health care costs, ${ }^{24-27}$ and is fatal in approximately $15 \%$ of patients. ${ }^{22}$ As a result, cost savings associated with switching to trastuzumab SC could be greater than those reported here. Using our estimate based on costs associated with HCP time and consumables only, multiplying the cost saving per cycle by the total number of treatment cycles given $(6,752)$ to all the patients with $\mathrm{eBC}$ and $\mathrm{mBC}$ treated with trastuzumab in New Zealand in 2012 (the most-recent set of complete PHARMAC special authority data; $n=460$ ), annual cost savings to the New Zealand Health Care System associated with a switch from trastuzumab IV infusion to trastuzumab SC injection could be more than half a million dollars (\$519,499 NZD).

The calculations made based on the results of the current study assume that the total cost of trastuzumab therapy 
to the New Zealand healthcare system would be equivalent whether the SC or IV formulation was used. These calculations could be influenced by the introduction of a biosimilar IV trastuzumab, but this is not expected to occur in New Zealand until at least the first half of 2019 (on the basis of clinical trial data timelines on clinicaltrials.gov and estimated regulatory review processes). On a patient-by-patient basis, the cost of trastuzumab therapy may vary, given that the dosage for trastuzumab IV is calculated based on patient bodyweight and trastuzumab SC is given as a single fixed dose. Nevertheless, on a population basis, overall drug costs are likely to be similar, although exact figures for the New Zealand setting remain to be determined, on the basis of negotiations around access and funding.

Another reason why the current data may underestimate the resource-use impact of switching from trastuzumab IV to the SC formulation is that only in-hospital direct medical costs were considered. This means that costs incurred by the patient either directly (eg, transport, parking) or indirectly (eg, time and loss of productivity for both patients and their caregivers) have not been quantified.

Patient preference, well-being, and quality of life were also not assessed in this study, but the PrefHer trial found that patients overwhelmingly preferred trastuzumab SC over the IV formulation. ${ }^{19}$ By far the most common reason cited by patients for this preference was that receiving trastuzumab as a SC injection saved time. Other reasons for preferring trastuzumab SC were less pain or discomfort and fewer side effects, the avoidance of problems associated with IV treatment (ie, venous access), and convenience. ${ }^{19}$ These findings suggest that switching to trastuzumab SC not only offers financial and logistical advantages to the health care system but is also perceived as a more-attractive treatment option by patients.

This study had a number of limitations, including the small sample size (particularly in the trastuzumab SC treatment group) and the administration of trastuzumab SC in only one of the two study centers. However, trastuzumab $\mathrm{SC}$ is not yet funded in New Zealand and is therefore not administered in routine clinical practice. As a result, the only available data on administration of trastuzumab SC was from consenting patients who were receiving trastuzumab SC at the single New Zealand center participating in the ongoing SafeHer trial. For both the SC and IV groups, it was not possible to report patient demographic data because the patient consent obtained applied only to observation and recording of tasks and times associated with trastuzumab administration and not access to patient medical files. For the IV group, it is likely that the patients treated were a relatively heterogeneous group because no selection was undertaken and any patient receiving trastuzumab was eligible (eg, eBC or $\mathrm{mBC}$ ). Patients in the SC group had only $\mathrm{eBC}$ and therefore it is possible that disease severity was a confounding factor because patients who were more unwell might have needed more HCP involvement. Despite these issues, the range of values obtained was relatively narrow, and expected time savings were comparable to those from international studies with broadly similar study design and objectives. It is possible that the results of this study cannot be generalized to centers other than the two that participated in this study. However, there are only six regional oncology centers in New Zealand, and $40 \%$ of New Zealand's cancer patients are treated at Auckland City Hospital, which contributed patients to this study. It should also be noted that in New Zealand there is no regional variation in drug costs because these are mandated nationally by PHARMAC, and unit costs for HCP and personnel time were derived from PHARMAC-specified national values. ${ }^{17}$ Therefore, it is likely that the findings will be relevant across the New Zealand health care setting.

The new SC formulation of trastuzumab represents a clinical opportunity to improve the efficiency of HER2-positive breast cancer treatment delivery by providing time and cost savings and the potential to modify pathways of patient care. This contributes to New Zealand Ministry of Health goals for increasing oncology treatment capacity and allowing treatment to be administered away from regional oncology centers. ${ }^{15}$ It should be noted that the time and equipment savings associated with a switch to trastuzumab SC are not a true financial saving but instead represent the value of the best available alternative use of a resource ("opportunity cost"). Fewer health care staff and infusion chairs would be needed to treat the same number of patients with trastuzumab SC, and this resource is then available to be redeployed in the treatment of other patients. Thus, at least in the short term, the main result of switching from trastuzumab IV to trastuzumab $\mathrm{SC}$ would be to ease pressure at capacity-constrained treatment centers.

\section{Conclusion}

Data from this New Zealand time and motion study provide an indication that switching from IV to SC administration of trastuzumab has beneficial effects on resource use, including HCP time and consumables needed to administer treatment. 
These reductions contribute to health care cost efficiencies, could help to address current capacity issues at New Zealand District Health Boards, and have the potential to influence how and where HER2-positive breast cancer treatment is delivered in the future.

\section{Acknowledgments}

PharmaMark was contracted to adapt UK methodology and data collection forms for use in New Zealand and conducted the study on behalf of Roche Products (New Zealand) Ltd. The authors would like to thank Chris Frampton for assistance with statistical analysis. Medical writing support was provided by Nicola Ryan, independent medical writer, funded by Roche Products (New Zealand) Ltd.

\section{Author contributions}

All authors made substantial contributions to data generation and analysis, drafting or critical revision of the manuscript, and approval for the final version to be published.

\section{Disclosure}

This study was funded by Roche Products (New Zealand) Ltd. Roche personnel contributed to the study design and data analysis, and participated in preparation of the study manuscript. SNR is a current employee of Roche Products (New Zealand) Ltd. RTN, VJH, and LCC declare that they have no competing interests. The authors report no other conflicts of interest in this work.

\section{References}

1. Cancer Society of New Zealand. Cancer statistics [webpage on the Internet]. Wellington, NZ: Cancer Society of New Zealand; [updated February 21, 2011]. Available from: http://www.cancernz.org.nz/ divisions/auckland/about/cancer-statistics. Accessed March 18, 2015.

2. Ministry of Health. Cancer: new registrations and deaths 2011 [webpage on the Internet]. Wellington, NZ: Ministry of Health; 2014 [updated October 14, 2014]. Available from: http://www.health.govt.nz/publication/cancer-new-registrations-and-deaths-2011. Accessed March 18, 2015 .

3. McKenzie F, Ellison-Loschmann L, Jeffreys M. Investigating reasons for socioeconomic inequalities in breast cancer survival in New Zealand. Cancer Epidemiol. 2010;34(6):702-708.

4. Roche. Data Sheet: Herceptin ${ }^{\circledR}$. Auckland, NZ: Roche Products (New Zealand) Ltd; 2014. Available from: http://www.medsafe.govt.nz/profs/ datasheet/h/Herceptininf.pdf. Accessed May 11, 2015.

5. Kruse GB, Amonkar MM, Smith G, Skonieczny DC, Stavrakas S. Analysis of costs associated with administration of intravenous singledrug therapies in metastatic breast cancer in a US population. J Manag Care Pharm. 2008;14(9):844-857.

6. Vescia S, Baumgärtner AK, Jacobs VR, et al. Management of venous port systems in oncology: a review of current evidence. Ann Oncol. 2008; 19(1):9-15.

7. Pettengell R, Davies AJ, Harvey VJ. Experience with an implantable venous access system for chemotherapy. N Z Med J. 1991;104(915): 284-285.
8. Launay-Vacher V. An appraisal of subcutaneous trastuzumab: a new formulation meeting clinical needs. Cancer Chemother Pharmacol. 2013;72(6):1361-1367.

9. Shepard HM, Jin P, Slamon DJ, Pirot Z, Maneval DC. Herceptin. In: Chernajovsky Y, Nissim A, editors. Therapeutic Antibodies. Berlin, Germany: Springer-Verlag Berlin Heidelberg; 2008:183-219.

10. Roche. Data Sheet: Herceptin ${ }^{\circledR}$ SC. Auckland, NZ: Roche Products (New Zealand) Ltd; 2014. Available from: http://www.medsafe.govt. nz/Profs/Datasheet/h/herceptinscinj.pdf. Accessed May 11, 2015.

11. Frost GI. Recombinant human hyaluronidase (rHuPH20): an enabling platform for subcutaneous drug and fluid administration. Expert Opin Drug Deliv. 2007;4(4):427-440.

12. Bookbinder LH, Hofer A, Haller MF, et al. A recombinant human enzyme for enhanced interstitial transport of therapeutics. $J$ Control Release. 2006;114(2):230-241.

13. Wynne C, Harvey V, Schwabe C, Waaka D, McIntyre C, Bittner B. Comparison of subcutaneous and intravenous administration of trastuzumab: a phase I/Ib trial in healthy male volunteers and patients with HER2-positive breast cancer. J Clin Pharmacol. 2013;53(2): $192-201$.

14. Ismael G, Hegg R, Muehlbauer S, et al. Subcutaneous versus intravenous administration of (neo)adjuvant trastuzumab in patients with HER2-positive, clinical stage I-III breast cancer (HannaH study): a phase 3, open-label, multicentre, randomised trial. Lancet Oncol. 2012;13(9):869-878.

15. Ministry of Health: Medical Oncology National Implementation Plan. 2012/13. Wellington, NZ: Ministry of Health; 2012 [updated October 10, 2012. Available from: http://www.health.govt.nz/publication/medicaloncology-national-implementation-plan-2012-13. Accessed March 18, 2015.

16. Burcombe R, Chan S, Simcock R, Samanta K, Percival F, Barrett-Lee P. Subcutaneous trastuzumab (Herceptin ${ }^{\mathbb{R}}$ ): A UK time and motion study in comparison with intravenous formulation for the treatment of patients with HER2-positive early breast cancer. Adv Breast Can Res. 2013;2(4):133-140.

17. Pharmaceutical Management Agency (PHARMAC). Cost Resource Manual. Wellington, NZ: PHARMAC; 2014. Available from: http:// www.pharmac.health.nz/assets/pfpa-v2-1-cost-resource-manual.pdf. Accessed March 18, 2015

18. De Cock E, Knoop A, Jakobsen EH, et al. Manual injection of subcutaneous trastuzumab vs intravenous infusion for HER2-positive early breast cancer: a time-and-motion study. European Cancer Congress 2013; 2013 Sep 27-Oct 1; Amsterdam, NL. Abstract \#P128.

19. Pivot X, Gligorov J, Müller V, et al; PrefHer Study Group. Preference for subcutaneous or intravenous administration of trastuzumab in patients with HER2-positive early breast cancer (PrefHer): an openlabel randomised study. Lancet Oncol. 2013;14(10):962-970.

20. Rule S, Collins GP, Samanta K. Subcutaneous vs intravenous rituximab in patients with non-Hodgkin lymphoma: a time and motion study in the United Kingdom. J Med Econ. 2014;17(7):459-468.

21. De Cock E, Pan I, Sandoval M, Millar D, Knoop A. Healthcare professionals' perceptions of the impact of switching from the intravenous to the subcutaneous formulation of trastuzumab. Ninth European Breast Cancer Conference; 2014 March 19-21; Glasgow, Scotland. Abstract\#42.

22. National Institute for Health and Clinical Excellence: Infection: Prevention and Control of Healthcare-Associated Infections in Primary and Community Care. Clinical Guideline: Methods, Evidence and Recommendations. London, UK: National Clinical Guideline Centre; 2012. Available from: http://www.nice.org.uk/guidance/cg139/ evidence/cg139-infection-control-full-guideline3. Accessed March 18, 2015.

23. Wilson M, Porter DJ, Maher A. Safety and efficacy of implanted central venous catheters during adjuvant treatment of breast cancer and in a general oncology population. American Society of Clinical Oncology 2011 Annual Meeting; Chicago, IL. J Clin Oncol. 2011;29(15 Suppl): Abstract \# 9124. 
24. Boersma RS, Schouten HC. Clinical practices concerning central venous catheters in haematological patients. Eur J Oncol Nurs. 2010;14(3): 200-204.

25. Rosenthal VD, Guzman S, Migone O, Crnich CJ. The attributable cost, length of hospital stay, and mortality of central line-associated bloodstream infection in intensive care departments in Argentina: A prospective, matched analysis. Am J Infect Control. 2003;31(8):475-480.

26. Worth LJ, Brett J, Bull AL, McBryde ES, Russo PL, Richards MJ. Impact of revising the National Nosocomial Infection Surveillance System definition for catheter-related bloodstream infection in ICU: reproducibility of the National Healthcare Safety Network case definition in an Australian cohort of infection control professionals. Am J Infect Control. 2009;37(8):643-648.
27. Public Health Agency of Canada. Preventing infections associated with indwelling intravascular access devices. Canada Communicable Disease Report - Supplement [webpage on the Internet]. Ottawa, ON: Public Health Agency of Canada; 1997 [updated November 8, 2002]. Available from: http://www.collectionscanada. gc.ca/webarchives/20071212094643/http:/www.phac-aspc.gc.ca/ publicat/ccdr-rmtc/97vol23/23s8/index.html\#. Accessed May 11, 2015 .

\section{Publish your work in this journal}

ClinicoEconomics \& Outcomes Research is an international, peerreviewed open-access journal focusing on Health Technology Assessment, Pharmacoeconomics and Outcomes Research in the areas of diagnosis, medical devices, and clinical, surgical and pharmacological intervention. The economic impact of health policy and health systems organization also constitute important areas of coverage. The manuscript management system is completely online and includes a very quick and fair peer-review system, which is all easy to use. Visit http://www.dovepress.com/testimonials.php to read real quotes from published authors.

Submit your manuscript here: http://www.dovepress.com/clinicoeconomics-and-outcomes-research-journal 